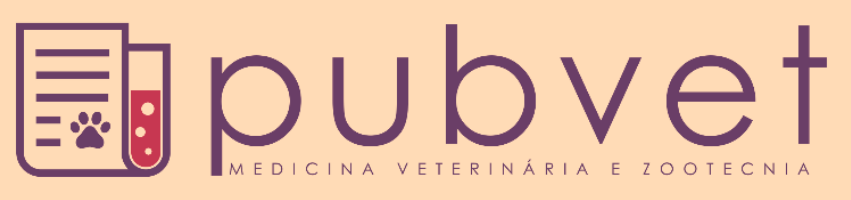

https://doi.org/10.31533/pubvet.v12n10a196.1-7

\title{
Estudo de anemias e policitemias registradas em cães de Joinville/SC de 2015 a 2017
}

\author{
Beatriz dos Santos Bello ${ }^{*} \bullet$, Carlos Eduardo Nogueira Martins ${ }^{2} \bullet$, Francielle Diane de \\ Medeiros $^{3}{ }^{\bullet}$, Michele Muller Fialkowski $^{3}{ }^{\circ}$, Pricilla Pozzatti ${ }^{4} \bullet$
}

${ }^{1}$ Acadêmica do Instituto Federal Catarinense. Araquari-SC Brasil. E-mail: beatriz.sbello@gmail.com

${ }^{2}$ Professor do Instituto Federal Catarinense, departamento de medicina veterinária. Araquari-SC Brasil.

${ }^{3}$ Colaboradoras do projeto proprietárias dos laboratórios Medivet e Badanievet respectivamente. Joinville-SC Brasil.

${ }^{4}$ Professora do Instituto Federal Catarinense orientadora do projeto, departamento de medicina veterinária. Araquari-SC Brasil.

*Autora para correspondência

RESUMO O diagnóstico de muitas doenças que acometem os animais possui auxílio de exames laboratoriais, pois estes podem demonstrar alterações significativas do organismo não vistas no exame físico. O hemograma possui todos os parâmetros para examinar as células sanguíneas dos vasos periféricos sendo estas classificadas como eritrócitos, leucócitos e plaquetas. É o exame mais utilizado na rotina clínica por sua praticidade, economia e utilidade clínica, o qual está dividido em eritrograma, leucograma e trombograma. Por ser um assunto de grande importância clínica, é necessário que haja um conhecimento adequado sobre os exames para que seja realizada a interpretação correta e o devido tratamento do animal. Para este fim foram coletados laudos de hemogramas de dois laboratórios de patologia clínica veterinária da cidade de Joinville - SC e a partir destes realizou-se triagens e separação em grupos. Primeiramente foram separados os exames que possuíam as informações relacionadas a sexo, raça e idade, totalizando 13286 exames. Posteriormente formou-se dois grupos, um com laudos de animais com anemia $(n=3294)$ e outro com policitemia $(n=2747)$. Dentro dos dois grupos foram criados 3 subgrupos, fazendo separação de todos os laudos em sexo, raça e idade. As idades foram separadas em três grupos: jovens menores que 1 ano, adultos entre 1 e 8 anos, idosos acima de 8 anos. As raças foram agrupadas nominalmente, sendo os sem raça definida (SRD) classificados como uma raça e ao fim das pesquisas foram discutidas as três raças que tiveram maior quantidade de laudos analisados. Terminadas todas as triagens fez-se o teste do quiquadrado através do programa estatístico R para obter uma análise descritiva dos dados. Por fim observou-se um predomínio de fêmeas, SRD e idosos tanto no grupo de anemias quanto de policitemias, porém, para ambos os grupos todos os subgrupos formados tiveram diferença entre si. Sendo assim sugere-se que haja um grupo de risco para anemias e policitemias porém são necessárias mais pesquisas acerca do assunto.

Palavras chave: anemia, idade, policitemia, raça, sexo

\section{Study of anemia and polycythemia recorded in dogs from Joinville/SC from 2015 to 2017}

ABSTRACT The diagnosis of many diseases that affect the animals has the aid of laboratory tests, as these can demonstrate significant changes of the organism not seen in the physical examination. The blood count has all the parameters to examine the blood cells of the peripheral vessels and these are classified as erythrocytes, leukocytes and platelets. It is the most commonly used exam in the clinical routine due to its practicality, economy and clinical utility, which is divided into erythrogram, leukogram and thrombogram. 
Because it is a subject of great clinical importance, it is necessary that there is adequate knowledge about the tests so that the correct interpretation and proper treatment of the animal is performed. For this purpose, hemoglobin reports were collected from two laboratories of veterinary clinical pathology in the city of Joinville - SC, and from these, triage and separation were performed in groups. Firstly, the exams that had the information related to gender, breed and age were separated, totaling 13286 exams. Two groups were later formed, one with reports of anemia $(n=3294)$ and one with polycythemia $(n=2747)$. Within the two groups, 3 subgroups were created, separating all the reports on sex, breed and age. The ages were separated into three groups: young than 1 year old, adults between 1 and 8 years old, aged over 8 years old. The races were grouped by name, being the non defined breed (SRD) classified as a race and at the end of the research the three races that had the highest number of analyzed reports were discussed. After all triages were completed, the chi-square test was performed through the statistical program $\mathrm{R}$ to obtain a descriptive analysis of the data. Finally, a predominance of females, SRD and elderly in both the anemia and polycythemia groups was observed, but for all the groups, all the subgroups formed had a difference between them. Therefore, it is suggested that there is a risk group for anemia and polycythemia, but further research is needed.

Keywords: age, anemia, breed, polycythemia, sex

\section{Estudio de anemias y policitemias registradas en perros de Joinville / SC de 2015 a 2017}

RESUMEN El diagnóstico de muchas enfermedades que afectan a los animales tiene ayuda de exámenes de laboratorio, ya que éstos pueden mostrar alteraciones significativas del organismo no vistas en el examen físico. El hemograma posee todos los parámetros para examinar las células sanguíneas de los vasos periféricos siendo estas clasificadas como eritrocitos, leucocitos y plaquetas. Es el examen más utilizado en la rutina clínica por su practicidad, economía y utilidad clínica, el cual está dividido en eritrograma, leucograma y trombograma. Por ser un asunto de gran importancia clínica, es necesario que haya un conocimiento adecuado sobre los exámenes para que se realice la interpretación correcta y el debido tratamiento del animal. Para este fin fueron recolectados laudos de hemogramas de dos laboratorios de patología clínica veterinaria de la ciudad de Joinville - SC y a partir de éstos se realizaron clasificaciones y separación en grupos. Primero fueron separados los exámenes que poseían las informaciones relacionadas con sexo, raza y edad, totalizando 13286 exámenes. Posteriormente se formaron dos grupos, uno con laudos de animales con anemia $(n=3294)$ y otro con policitemia $(n=2747)$. En los dos grupos se crearon 3 subgrupos, separando todos los laudos en sexo, raza y edad. Las edades fueron separadas en tres grupos: jóvenes menores de 1 año, adultos entre 1 y 8 años, ancianos mayores de 8 años. Las razas fueron agrupadas nominalmente, siendo los cruzados como una raza y al final de las investigaciones fueron discutidas las tres razas que tuvieron mayor cantidad de laudos analizados. Terminadas todas las clasificaciones se hizo la prueba del chi-cuadrado a través del programa estadístico R para obtener un análisis descriptivo de los datos. Por fin se observó un predominio de hembras, criollas y ancianos tanto en el grupo de anemias y de policitemias, pero para ambos grupos todos los subgrupos formados tuvieron diferencia entre sí. Siendo así se sugiere que haya un grupo de riesgo para anemias y policitemias pero son necesarias más investigaciones sobre el asunto.

Palabras clave: anemia, edad, policitemia, raza, sexo

\section{Introdução}

A anemia, que é a redução do volume de células vermelhas no sangue, é uma das alterações mais avaliadas no hemograma e está frequentemente associada a doenças hematológicas e não hematológicas como infecções, neoplasias, insuficiência renal dentre outras. Não é caracterizada como uma doença, e sim um estado patológico, logo, sua identificação tem grande colaboração para chegar a um diagnóstico (Lacerda, 2015; Couto, 2010). Em 
contrapartida, a policitemia é o aumento na quantidade de células sanguíneas (Thrall et.al., 2006).

O diagnóstico de muitas doenças que acometem os animais possui auxílio de exames laboratoriais, pois estes podem demonstrar alterações significativas do organismo não vistas no exame físico (Dias et al., 2013). O hemograma possui todos os parâmetros para examinar as células sanguíneas dos vasos periféricos. Essas células são classificadas como eritrócitos ou hemácias (células vermelhas), leucócitos (células brancas) e plaquetas (Perez \& Ristow, 2014).

Para avaliação e classificação da anemia, existem três parâmetros. $\mathrm{O}$ primeiro é baseado no tamanho das hemácias e teor de hemoglobina avaliando-se o Volume Corpuscular Médio (VCM) e a Concentração de Hemoglobina Corpuscular Média (CHCM). O segundo método avaliativo ocorre através da resposta da medula óssea para verificar se a anemia é regenerativa ou não e a terceira classificação leva em conta a fisiopatogenia, baseando-se na disfunção primária. Esta última propicia uma base ao clínico para o diagnóstico da possível causa da anemia (Thrall et al., 2006). Dentre os índices hematimétricos o VCM é o mais utilizado na avaliação das anemias, associado às análises morfológicas das hemácias que auxiliam na classificação (Grotto, 2009).

As policitemias, por sua vez, podem ser classificadas como relativas ou absolutas. A policitemia relativa pode ocorrer por diminuição do volume plasmático, redistribuição eritrocitária ou contração esplênica. Já a policitemia absoluta, também chamada de policitemia vera, pode ser dividida em primária e secundária, sendo que a primária se desencadeia por distúrbios mieloproliferativos e a secundária por aumento de eritropoietina (Soto et al., 2008).

A idade pode ter relação nas anemias pois na fase senil a medula óssea se torna fibrosada com difícil expansão, por isso os animais idosos possuem maior dificuldade de resposta frente a anemias (Lopes, et al., 2007). Além disso, cães idosos possuem redução do fluxo sanguíneo dos rins, deixando-os com tamanho menor e dessa forma provocando a uma Insuficiência Renal Crônica (IRC). A maior parte dos pacientes com DRC encontra-se anêmica pela deficiência de eritropoietina que é produzida neste órgão (Hoskins, 2008; Stockham \& Scott, 2011). A policitemia nessa faixa etária também é justificável, visto que animais idosos tendem a desidratar com mais facilidade. (Lopes, et al., 2007). Em contrapartida, Lund (2007) observou em seu estudo que animais adultos, com idade média de 3,1 anos, apresentaram mais quadros anêmicos que animais idosos.

Ao avaliar as raças, nota-se que cães sadios da raça Akita e Shiba possuem valores de VCM abaixo de cães de outras raças, ocorrendo o mesmo com cães asiáticos como Chow-chow e Sharpei (Stockham \& Scott, 2011). Também há relatos que cães da raça poodle possuem uma predisposição a anemia hemolítica não-esferocitária, um distúrbio idiopático herdado com caráter autossômico dominante (Fighera, 2007). Além disso deve-se levar em consideração que $48,5 \%$ da população canina brasileira é caracterizada como raças de pequeno porte (IBGE, 2007), e também através de um estudo realizado em Teresina é possível observar que grande parte da população tem preferência por cães SRD (Quessada et al., 2014). Dessa forma, é possível que a maior parte das raças consultadas em clínicas, consultórios e hospitais sejam de pequeno porte ou SRD.

No que diz respeito ao sexo, Lund (2007) demonstrou que não há relação de machos e fêmeas com a ocorrência de anemia. Apesar disso, Crafts (1948) avaliou o efeito do estrogênio na medula óssea de cadelas adultas e comprovou que doses altas deste hormônio provocam anemia.

Por ser um assunto de grande importância clínica, é necessário que haja um conhecimento adequado sobre este para que seja realizada a interpretação correta dos exames e o devido tratamento do animal. Sendo assim, o objetivo do estudo foi avaliar a relação de anemias e policitemias com sexo, raça e idade.

\section{Material e métodos}

Em contato prévio com os laboratórios BADANIEVET e MEDIVET da cidade de Joinville-Santa Catarina foram solicitados os laudos dos hemogramas realizados em cães nos anos de 2015 a 2017 os quais foram cedidos, totalizando cerca de 20.000 exames.

Foi realizada uma triagem para separar primeiramente os laudos que possuíam todos os dados necessários para a pesquisa. Dos exames aptos para este estudo, foi realizada uma separação de laudos que se estruturou dois grandes grupos: $o$ primeiro grupo, que se constituiu de animais que apresentaram baixa concentração de hemácias, quadro caracterizado como anemia e o segundo 
grupo, que apresentou indivíduos que com alta concentração de hemácias, quadro esse conhecido como policitemia. Para cada grande grupo, os laudos foram agrupados segundo raça, sexo e idade. No caso particular da idade, a separação foi realizada segundo Schalm et al. (2000) conforme demonstrado no quadro 1 .

Quadro 1. Valores de referência utilizados na separação dos laudos quanto a idade, conforme descrito por Schalm et al. (2000).

\begin{tabular}{|c|c|c|c|c|c|}
\hline Hematócrito & $\begin{array}{c}<3 \\
\text { meses }\end{array}$ & $\begin{array}{c}3 \text { a } 6 \\
\text { meses }\end{array}$ & $\begin{array}{c}6 \text { a } 12 \\
\text { meses }\end{array}$ & $\begin{array}{c}1 \text { a } 8 \\
\text { anos }\end{array}$ & $\begin{array}{c}>8 \\
\text { anos }\end{array}$ \\
\hline$\%$ & $26-39$ & $34-40$ & $40-47$ & $37-55$ & $38-47$ \\
\hline
\end{tabular}

Quando avaliados pela idade, foram considerados jovens os animais abaixo de 1 ano, adultos entre 1 e 8 anos e idosos acima de 8 anos conforme estipulado pelos autores. Para animais que se encaixaram em dois subgrupos de idade (por exemplo, animais de 6 meses se enquadraram na categoria de 3-6 meses e 6-12 meses) juntou-se as duas referências que corresponderam a essas idades (por exemplo, para animais de 6 meses foi utilizada a referência de $34-47 \%$ de hematócrito, considerando um intervalo de 3 a 12 meses).

No que diz respeito a separação por raças, estas foram agrupadas nominalmente, sendo os sem raça definida (SRD) classificados como uma raça. A discussão foi realizada utilizando-se as três raças que tiveram maior quantidade de laudos analisados. Raças que apareceram 10 vezes ou menos nos laudos foram classificadas em um grupo denominado "outras raças".

Por fim formaram-se grupos da seguinte maneira: um grupo de anêmicos com subgrupos de sexo (macho e fêmea), idade (jovem, adulto e idoso) e raça. Outro grupo de policitêmicos com subgrupos de sexo (macho e fêmea), idade (jovem, adulto e idoso) e raça. Cada subgrupo criado foi independente do outro, ou seja, o total de laudos de animais que apresentaram anemia entrou nos 3 subgrupos e o mesmo acorreu para policitemia.

Foi realizada uma análise estatística dos dados para avaliar a relação da anemia e da policitemia com a raça, a idade e o sexo, utilizando-se o teste de qui-quadrado através do programa estatístico $\mathrm{R}$.

\section{Resultados e discussão}

Foram analisados 19.958 exames os quais após a primeira triagem chegou-se a um total de 13.286 laudos completos, sendo que destes, 3.294 apresentaram anemia e 2.747 apresentaram policitemia.
Sexo

O grupo correspondente a anemia obteve um resultado de $61,6 \%$ fêmeas e 38,4\% machos. Já no grupo relacionado a policitemia chegou-se ao resultado de $64,3 \%$ fêmeas e $35,7 \%$ machos, ambos os grupos com diferença significativa ( $\mathrm{p}<$ 0,001 ) pelo teste do qui-quadrado, conforme mostra a figura 1.

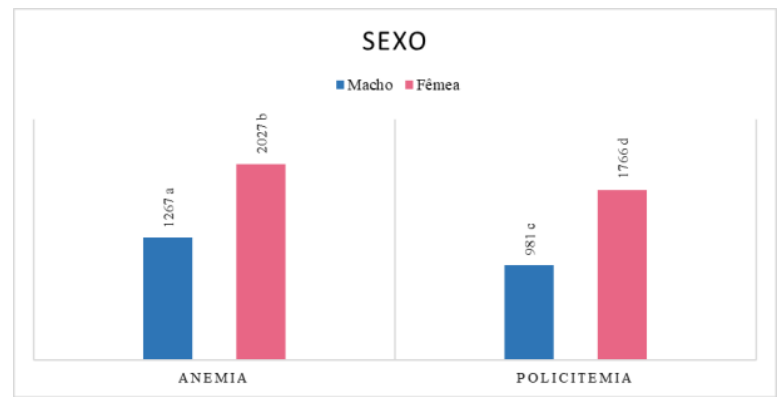

Figura 1. Gráfico de valores obtidos de pacientes com alterações hematológicas e comparação entre os sexos com relação a anemia $(n=3294)$ e policitemia $(n=2747)$.

*Valores seguidos de letras distintas apresentaram diferença estatística entre si pelo teste do qui-quadrado ( $p<0,001)$.

Existem relatos da associação dos altos níveis de estrógeno com o aparecimento de anemia em cadelas adultas (Crafts 1948) e também a interferência da Prostaglandina $2 \alpha$ que inibe a eritropoiese (Lacerda, 2015). Observando-se a população canina brasileira constata-se, através de pesquisas, que $81,3 \%$ dos animais não são castrados (IBGE, 2007), fator que pode causar interferências medulares. Porém, o presente estudo não utilizou histórico clínico dos pacientes, sugere-se, portanto, a avaliação do uso de contraceptivos em animais quanto ao aparecimento de processos anêmicos e também o histórico de castração.

\section{Idade}

Observou-se que no grupo de animais com anemia houve maior número de animais idosos $(51,7 \%)$, seguida de animais adultos $(34,8 \%)$, sendo que os jovens apareceram em menor quantidade $(13,5 \%)$.

Em contrapartida no grupo de animais com policitemias o subgrupo de idosos continua em primeiro lugar $(52,5 \%)$ seguido de animais jovens $(26,6 \%)$ e em terceiro lugar ficaram os animais adultos (21,2\%) conforme figura 2. Houve diferença estatística significativa entre as idades dos dois grupos $(\mathrm{p}<0,001)$.

Os resultados obtidos no presente estudo concordam com os autores Lopes, et al. (2007) que 
demonstraram que animais idosos possuem a medula óssea fibrosa com difícil expansão, por isso não respondem de forma eficaz frente a um quadro de anemia. Os mesmos autores também relatam que animais senis são mais propensos a desidratação, fator que contribui para ocorrência de policitemia relativa. Porém, existe uma contraposição com o estudo feito por Lund (2007), que demonstrou que os animais adultos na faixa de 3 anos apresentaram mais casos de anemia em relação aos cães idosos.

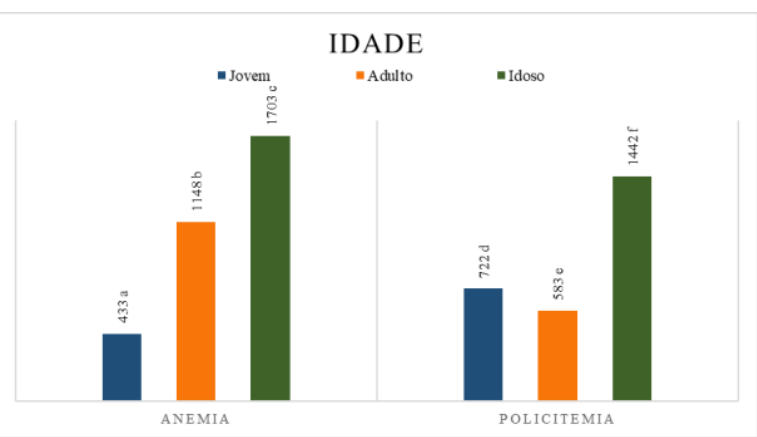

Figura 2. Gráfico de valores obtidos de pacientes com alterações hematológicas e comparação entre as idades com relação a anemia $(n=3294)$ e policitemia $(n=2747)$

*Valores seguidos distintas apresentaram diferença estatística entre si pelo teste do qui-quadrado $(\mathrm{p}<0,001)$.

Raça

$\mathrm{O}$ quadro 2 apresenta o nome e total de raças dos laudos analisados com anemias e policitemias.

No grupo relacionado a anemias, as raças predominantes foram SRD $(37,3 \%)$, Poodle $(8 \%)$ e Pinscher $(5,5 \%)$ em ordem decrescente, conforme apresentado na figura 3 . Através dos dados estatísticos foi constatada diferença entre os 3 grupos $(\mathrm{p}<0,001)$.

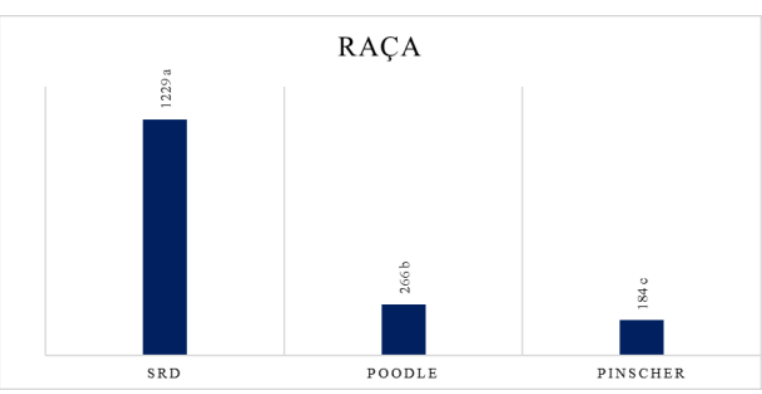

Figura 3. Gráfico de valores obtidos de pacientes com alterações relacionadas a anemia $(n=3294)$ e a diferença das três raças que apareceram em maior número nesse grupo. *Valores seguidos de letras distintas apresentaram diferença estatística entre si pelo teste do qui-quadrado ( $<<0,001)$.

A população de cães SRD é mais volumosa em relação a cães de raça, podendo justificar seu aparecimento em maior número nos resultados (Quessada et al., 2014). Já a raça Poodle ficou em segundo lugar na quantidade de laudos que apresentaram anemia, concordando com Fighera (2007) que relata que cães da raça poodle possuem uma predisposição a quadros anêmicos. Além disso, há relatos de que existe preferência de grande parte da população por cães de pequeno porte (IBGE, 2007), o que justifica o grande número de casos encontrados.

Quadro 2. Quadro de valores obtidos de pacientes com alterações relacionadas a anemia $(n=3294)$ e policitemia $(\mathrm{n}=2747)$ separados de acordo com a raça.

\begin{tabular}{|c|c|c|c|}
\hline \multicolumn{2}{|c|}{ Anemia } & \multicolumn{2}{|c|}{ Policitemia } \\
\hline Raça & Total & Raça & Total \\
\hline SRD & 1229 & SRD & 773 \\
\hline Poodle & 266 & Yorkshire & 385 \\
\hline Pinscher & 184 & Poodle & 333 \\
\hline Labrador & 159 & Pinscher & 275 \\
\hline Shih tzu & 152 & Shih tzu & 146 \\
\hline Yorkshire & 142 & Lhasa apso & 114 \\
\hline Cocker & 105 & Daschund & 82 \\
\hline Pit bull & 101 & Maltês & 70 \\
\hline Rotweiller & 85 & Schnauzer & 68 \\
\hline Schanuzer & 81 & Bulldog & 64 \\
\hline Lhasa apso & 80 & Labrador & 49 \\
\hline Boxer & 69 & Pastor alemão & 47 \\
\hline Daschund & 69 & Boxer & 38 \\
\hline Pastor alemão & 51 & Pit bull & 34 \\
\hline Chow chow & 41 & Beagle & 27 \\
\hline Golden & 41 & Pequenês & 26 \\
\hline Pequinês & 41 & Spitz & 22 \\
\hline Beagle & 35 & Cocker & 15 \\
\hline Pug & 32 & Pug & 15 \\
\hline Akita & 31 & Chow chow & 15 \\
\hline Maltês & 31 & Teckel & 13 \\
\hline Sharpei & 31 & Golden retrivier & 12 \\
\hline Dálmata & 29 & Fox paulisitnha & 11 \\
\hline Border Collie & 24 & Border collie & 11 \\
\hline Bulldog & 17 & Scotich & 11 \\
\hline Bull terrier & 15 & Outras & 91 \\
\hline Scotisch & 13 & & \\
\hline American & 13 & & \\
\hline Fox Paulistinha & 11 & & \\
\hline Outras & 116 & & \\
\hline
\end{tabular}

Já no grupo das policitemias, as raças predominantes foram SRD $(28,1 \%)$, Yorkshire $(14 \%)$ e Poodle $(12,1 \%)$ em ordem decrescente conforme figura 4 . Através dos dados estatísticos foi constatada diferença entre os 3 grupos ( $p<$ $0,001)$. 
A mesma justificativa para o aparecimento de anemias em cães SRD pode ser feita ao avaliar a quantidade de laudos encontrados com policitemia. O fato da população brasileira ter preferência para as raças de pequeno porte (IBGE, 2007) e para SRD, levam ao maior aparecimento de casos.

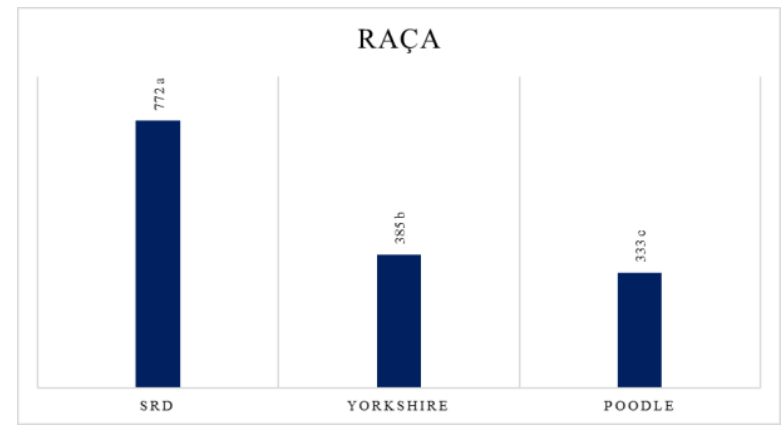

Figura 4. Gráfico de valores obtidos de pacientes com alterações relacionadas a policitemia $(n=2747)$ e a diferença das três raças que apareceram em maior número nesse grupo. *Valores seguidos de letras distintas apresentaram diferença estatística entre si pelo teste do qui-quadrado ( $<<0,001)$.

Dessa forma, pode não existir necessariamente uma predisposição dessas raças a quadros de anemias e policitemias, porém esses animais aparecem em primeiro lugar devido a grande população existente. Sugere-se mais estudos no que diz respeito a anemias e policitemias relacionado a raças.

\section{Conclusão}

Animais idosos possuem propensão a desenvolver alterações hematológicas por razões fisiológicas da idade.

$\mathrm{O}$ aparecimento em maior quantidade de raças de pequeno porte e SRD pode ser devido a preferência dos proprietários por animais com essa característica.

Fêmeas podem ter maiores alterações do que machos pela influência hormonal.

Porém os estudos são escassos nessa área, sendo assim, é importante a realização mais trabalhos a fim de se obter resultados mais concretos.

\section{Referências}

Couto, C. G. 2010. Anemia. In: Nelson, R. W. et al. Medicina interna de pequenos animais (4 ed.) Elsevier, Rio de Janeiro, Rio de Janeiro, Brasil.
Crafts, R. C. 1948. The effects of estrogens on the bone marrow of adult female dogs. Blood jornal, 3, 276-285.

Dias, C. S., Lima, I. T., Volpato, J., Weinert, N. C., Mattoso, C. R. S. \& Saito, M. E. 2014. Levantamento de exames laboratoriais e casuística de anemia em cães e gatos atendidos no hospital de clínica veterinária do CAVUDESC no ano de 2013. UDESC em ação, 8, $85-100$.

Fighera F. A. 2007. Anemia hemolítica em cães e gatos. Acta Scientiae Veterinariae. 35, 264266.

Grotto, H. Z. W. 2009. O hemograma: importância para a interpretação da biópsia. Revista Brasileira de Hematologia e Hemoterapia, 3 , 178-182.

Hoskins J. D. 2008. Sistema urinário. In: Hoskins J. D. (2 ed.) Geriatria e gerontologia do cão e gato. Roca, São Paulo, São Paulo, Brasil.

IBGE 2007. Pesquisa domiciliar sobre cães $e$ gatos: Humanização e padrões de consumo. Rio de Janeiro.

Lacerda, L. A. 2015. Anemias: Avaliação clínica e laboratorial. In: Jericó, M. M., Neto, J. P. A. e Kogiga M.M. (1 ed.) Tratado de medicina interna de cães e gatos. Roca, Rio de Janeiro, Rio de Janeiro, Brasil.

Lopes, S. T. A., Biondo A. W. \& Santos, A. P. 2007 Manual de patologia clínica veterinária (3 ed.) Santa Maria, Rio Grande do Sul, Brasil.

Lund E. 2013. Population analyses of anemia in pets. Data savant, 807, 16-24.

Perez, A. \& Ristow, L. 2014 Avaliação do hemograma e sua aplicação clínica parte 1 . Vetscience Magazine, 4, 14-15.

Quessada, A. M., Barbosa, E. L., Nunes, J. A. R., Oliveira, F. S., Último, A. P. \& Sugauara, E. Y. 2014. Perfil de proprietários de cães no município de Teresina (Brasil). Arquivos de Ciências Veterinárias e Zoologia da UNIPAR, 17(3), 173- 175.

Schalm, O. W., Weiss, D. J. \& Wardrop, K. J. 2000. Veterinary hematology (5 ed.) WileyBlackwell, Ames, Iowa, USA.

Soto, J. C. H., Oliveira, R. G., Menegueti, V. C. \& Sacco, S. R. 2008. Policitemia e eritrocitose em animais domésticos revisão de literatura. 
Revista científica eletrônica de medicina veterinária,1679-7353, 6 (11), 1-7.

Stockham, S. L. \& Scott, M. A. 2011. Eritrócitos. In: Stockham, S. L. e Scott, M. A. 2 ed. Fundamentos de patologia clínica veterinária. Guanabara Koogan, Rio de Janeiro, Rio de Janeiro, Brasil.

Thrall, M. A. 2006. Classificação e diagnóstico de anemia ( 1 ed.) In: Thrall, M. A. et al.
Hematologia e bioquímica clínica veterinária. Roca, São Paulo, São Paulo, Brasil.

Recebido: 2 agosto, 2018 .

Aprovado: 29 agosto, 2018

Publicado: 29 outubro, 2018

Licenciamento: Este artigo é publicado na modalidade Acesso Aberto sob a licença Creative Commons Atribuição 4.0 (CC-BY 4.0), a qual permite uso irrestrito, distribuição, reprodução em qualquer meio, desde que o autor e a fonte sejam devidamente creditados. 